\title{
Features of an e-learning environment which promote critical and creative thinking: choice, feedback, anonymity, and assessment
}

\author{
Michał Jasieński \\ Center for Innovatics, \\ Nowy Sacz Business School - National-Louis University, \\ ul. Zielona 27, 33-300 Nowy Sacz, Poland \\ E-mail: jasienski@post.harvard.edu
}

\begin{abstract}
I discuss features that are important for creative and critical thinking which should be recreated in e-learning applications. Anonymity maximises chances for development of creativity and for objective and accurate assessment. I also describe a 'quadruple anonymity' system implemented at Nowy Sacz Business School - National-Louis University in Poland, the goal of which is to improve objectivity of thesis evaluation by referees. E-learning environment is ideal for implementing functionalities which make choice, feedback, and controlled anonymity easily available to the users to be effective, feedback should be appropriately timed, incremental, impartial, and impersonal. Evaluation of student or employee performance or of proposed ideas or solutions should rely on explicitly stated quantitative criteria, developed along well thought-through measurement scales and utilising proper descriptive statistics and visualisation methods. A description of the nominal group heuristic method is provided as an example of a heuristic method which relies on creativity, anonymity and unbiased evaluation.
\end{abstract}

Keywords: anonymity; assessment; choice; creativity; e-learning; evaluation; feedback; heuristics; innovation; methodology; nominal group; quantitative methods; trust.

Reference to this paper should be made as follows: Jasieński, M. (2014) 'Features of an e-learning environment which promote critical and creative thinking: choice, feedback, anonymity, and assessment', Int. J. Continuing Engineering Education and Life-Long Learning, Vol. 24, Nos. 3/4, pp.237-251.

Biographical notes: Michał Jasieński holds an MSc from the Jagiellonian University and MA and PhD from Harvard University (Graduate School of Arts and Sciences). He has led projects within the 'The Creator of Innovativeness' governmental programme and established the Center for Innovatics. He is a Senior Researcher in the project 'Reinvent: Transforming SMEs in Creative Sectors through Business Model Innovation' (2013-2016), within the Marie Curie IAPP programme. He serves as Innovation Editor of the Journal of Entrepreneurship, Innovation, and Management. $\mathrm{He}$ is the co-Founder and Vice-President of the Salus Publica Foundation for Public Health and member of the board of the Kyoto-Kraków Foundation.

This paper is a modified and expanded version of a paper entitled 'Rules for constructing e-learning tools which foster independent, critical and innovative thinking' presented at 'Theoretical and Practical Aspects of Distance Learning: E-Learning for Societal Needs', Cieszyn, Poland, 15-16 October 2012. 


\section{Introduction}

As Amabile (1998) pointed out, in organisations 'creativity gets killed much more often than it gets supported' (see also Torr, 2008). Davila et al. (2005) referred to obstacles to innovativeness as 'organisational antibodies that kill off good ideas'. Will anything change if more of our organisational activities are moved to the virtual domain? What should we then pay attention to when designing e-learning tools that are supposed to assist, foster, nourish and/or stimulate innovative thinking (see e.g., a very interesting study of IBM managers by Lewis and Orton, 2000)? Is it at all possible to facilitate creativity using IT tools which, after all, cannot provide users with literal personal touch. If designed logically, can such tools compensate for this fundamental deficiency with appropriately designed functionalities, providing a form of guidance and knowledge sharing (see Smyrnova-Trybulska and Grudzień, 2009; Smyrnova-Trybulska and Stach, 2012; Stach and Smyrnova-Trybulska, 2012)?

In contrast to live creativity sessions which usually are (and, indeed, should be) characterised by 'creative' disorder, e-learning tools must rely on stimulating the users or participants by mechanisms of different nature. Paradoxically, creativity also needs discipline in thinking, not only free-flowing chaos. The process of implementing a heuristic method in software necessitates increased precision in designing the overall flow of procedures and clarity of instructions. Chang's (2011) concept of 'virtual teams with anonymity and structured interactions' emphasises order and structure underlying human virtual interaction, as necessary conditions for successful creativity. When Davila et al. (2005) set out to demystify innovativeness, they emphasised that solid, 'hardcore', management skills and explicitly applied metrics are essential to an organisation's innovativeness. However, Macfadyen and Dawson (2012) make a strong point that even developing detailed analytics is not a guarantee that e-learning organisations can make use of them!

Heuristic methods are never applied in vacuum, separated from the pressures of human personalities, from organisational culture and from administrative procedures (see Trompenaars, 2007). It is very important that basic administrative functions governing access, choice, anonymity, feedback, and assessment, be designed with utmost care. Such functionalities are not less important than the features which comprise the raison d'être of a given software or online application, i.e., those which provide the higher, 'intellectual' level of functionality (the ideation stage; see e.g., Daly et al., 2012).

Without these basic, 'physiological', features operating smoothly, effectiveness of e-learning tools, no matter how sophisticated, may be seriously compromised (see also Gilbert et al., 2007). The devil, as usual, is in the prosaic details. In this paper, I focus on such details which are basic features of an online system for innovative thinking, remembering that 'the bulk of the causes of low quality and low productivity belong to the system' and it is not the fault of the employees (Deming, 1982).

\section{Managing access and administrative privileges}

Clearly formulated procedures for deciding who and when should perform certain administrative activities are necessary for the proper functioning of online tools. The system must allow convenient ways for assigning who divides the group into teams, who has the right to post answers and who and when evaluates the proposed ideas. This issue 
is particularly important in the case of group activities, when e.g., a group of students, together with their section leader, form a profile for conducting a brainstorming session. Similarly, a team of employees may log in together to conduct a session according to the nominal group protocol (see below) to develop a new product. Sometimes, administrative privileges may form a hierarchical structure, with several teams (each led by its team leader) working independently on the same project, with departmental committee evaluating the proposed solutions.

An e-learning module dedicated to managing access may also maintain other functions, such as posting reminders about upcoming deadlines of successive rounds of heuristic session or controlling (or stimulating) submissions of proposed solutions or evaluations and feedback (see also Palmer and Holt, 2010).

\section{Managing choice: a case of 'two-sided selection' for thesis advisers and advisees}

It is a truism to say that creativity requires freedom of choice, of associations, or of selection of metaphors etc. It begins with choosing right people to work with, both when optimising the composition of a team of employees (Chi and Chen, 2009) or students (Krass and Ovchinnikov, 2006), selecting academic courses (e.g., Mockus, 2010), and when entering a mentor-pupil system. The thesis-writing process is such a situation and an e-learning environment should accommodate all the needs of both parties with respect to exercising choice. I describe here a system implemented at Nowy Sacz Business School - National-Louis University (WSB-NLU) in southern Poland.

Thesis adviser should be able to pick his or her advisees from among those students who expressed interest in his or her profile of topics. Students do not know who else applied to a particular adviser's group, so, if they are not chosen, they cannot know who outcompeted them and what specific criteria of choice were used by that faculty member.

Students have several options for tipping the adviser's decision in their favour: they can write a short cover letter with justification (directed to that particular adviser), and they can (but it is not obligatory) reveal their average grade and grades in those courses which are relevant or in which they performed well. Importantly, thesis adviser cannot obtain information about the applying advisee's grades.

Each student may apply at the same time, and prepare individualised 'portfolios' of information, to four thesis advisers, ranked in the order of the student's preference. If there are still vacant spots on the adviser's list, the system will fill them up at two additional rounds. First, it will add students who selected a given adviser at the lower position than rank 1 and the adviser has another window of opportunity to exercise his or her choice, by selecting among these students. At the end of the process, the system will fill all the empty spots by assigning randomly to advisers those students who either forgot or chose not to participate in the selection process.

\section{Managing anonymity: controlled and available whenever needed}

Providing and maintaining anonymity is an important component of various creative-thinking procedures since it maximises the chance for unconstrained creativity and/or objective evaluation of other people's views. When the need to protect personal 
image is removed, one's creativity may be greatly improved. de Bono's (1992) six thinking hats method serves the same purpose, when each participant's personal image and views are hidden behind a coloured hat metaphorically worn during the debate. Also, the referees are freed from the risk of interpersonal conflicts between them and the authors of ideas or, as can be seen in Section 4.1, between them and the authors' advisers. This effect is especially relevant in institutions with hierarchical and formal organisational cultures (Jasieński and Rzeźnik, 2012).

In live settings attempts at protecting privacy (at certain points during debates or team activities) may be considered as expression of lack of trust. In contrast, when anonymity is simply a pre-programmed, built-in feature of an automatic system, it may be managed more effectively and it is more readily accepted by the participants (see also: Anwar and Greer, 2012; Yong, 2008).

\section{1 'Do not ask-do not tell': a case of quadruple anonymity in thesis reviewing}

The system of managing submission of Bachelor's and Master's theses and obtaining reviews and grades for them may serve as a good illustration of the role of anonymity in influencing human interactions in the academic context. The Faculty of Entrepreneurship and Management at WSB-NLU introduced in 2009 a set of online procedures which guarantee freedom from any form of peer pressure that may be felt by the student, the reviewer and thesis adviser, all involved in the process of evaluating the submitted thesis. A statistical analysis of grades given by reviewers of over 3,000 theses showed that such variables as gender of the student, gender and academic rank of the thesis adviser, and gender and academic rank of the reviewer, had detectable and statistically significant impacts on the grades that students received for their theses (M. Jasieński, unpublished data). Introduced procedures were meant to remove such effects and establish fairness and trust (Jasieński, 2007).

The system is characterised by four-directional (quadruple) anonymity and is based on the following conditions:

1 The student does not know the identity of the thesis reviewer - this condition frees the reviewer from any form of stress related to knowing student's expectations with respect to the grade. The reviewer's name is never revealed to the student by the employees managing the faculty's administrative office. The content of the review, including the partial scores and the text of the review, is sent to the student's profile in the intranet before the thesis defence date.

2 Thesis adviser does not know the identity of the thesis reviewer - this condition frees the reviewer from any form of stress related to knowing the adviser's expectations with respect to the grade. Since most of the time the thesis adviser is the reviewer's colleague, and often is lower in academic ranking, such peer pressure could be, and indeed was observed to be, quite intense and detectable. As described above, the reviewer's name is never revealed to the thesis adviser by the administrators.

3 Thesis reviewer does not know the identity of the student - this condition frees the student from any form of lack of objectivity on the part of the reviewer related to knowing student's gender or academic quality. In other words, it protects the student from potential sexism and elitism sometimes exhibited by the reviewers (see also 
Boaler et al., 2011). The reviewer receives either the electronic (pdf) or a printed version of the thesis, either of which has the student's name (and also the page with acknowledgements) removed by the IT system.

4 Thesis reviewer does not know the identity of the thesis adviser - this condition protects the student from the negative effects of potential pre-existing conflicts between the academic colleagues who serve as the reviewer and adviser. The reviewer's low evaluation may be used as a form of punishment directed against the adviser, with the student author being, of course, the casualty of such academic conflicts. The reviewer's version of the thesis has the adviser's name removed by the IT system.

The online environment makes implementation of these procedures relatively easy, given that a customised system has been created and that the faculty members and administration agree with the system's objectives. The sense of freedom and independence that is the desired outcome of the above listed procedures are prerequisites for creativity in generating ideas and honesty of judgment in evaluating them.

\subsection{Anonymity based on trust: course evaluations}

Although I do not intend to discuss the strategies of constructing the actual questions used in course evaluation, it is worth emphasising that assessment of teaching quality should utilise, as much as possible, quantitative criteria. They should be definable by the user, should utilise several available measurement scales (from traditional to various versions of the Likert scale) and should offer a palette of descriptive statistics. An assessment module (see Section 7), an integrated set of procedures used for evaluating the quality of ideas generated by various heuristic methods, may also be adapted, with appropriate modifications, for the purpose of course evaluation.

However, with online course evaluations, in contrast to anonymously filled out paper questionnaires, there is an issue of trust. Students' reservations about the system's (or rather college administrators') honesty in upholding the promise of online anonymity may affect the objectivity of their evaluations. When personalised intranet profiles are used for completing the course evaluation, student's identity is, in principle, easily recoverable, and any evaluating institution must establish a strong image of trustworthiness, to ensure functioning of a reliable evaluation system (Jasieński, 2007).

\section{Managing feedback: regulated criticism helps creativity}

Importance and role of feedback in education have been emphasised in many studies (see e.g., Handley and Williams, 2011; Krause et al., 2009; Thoms, 2011; Tsai, 2010). For example, feedback is essential for an important concept of self-regulated learning (Butler and Winne, 1995; Nicol and Macfarlane-Dick, 2006). At the same time, one should not forget that feedback should flow both from and to all stakeholders in the educational process, i.e., students, teachers, but also administrators and educational technologists (Jara and Mellar, 2010) - they should all have proactive rather than reactive roles, i.e., both as users and generators of feedback (Nicol and Macfarlane-Dick, 2006). Here, I would like to point to some fundamental features of feedback that should be considered by designers of e-learning systems. 


\subsection{Appropriately-timed feedback nourishes motivation for creativity}

On one hand, research on human decision making shows that we have a remarkable ability to form judgments that are based on information gathered almost intuitively. Feedback based on such immediate responses could be very perceptive. Immediacy of evaluations may allow the participants to benefit from a continuous stimulation, maintaining interest in the topic. Often, attempts at consciously taking into account all the relevant information result in 'analysis paralysis' and lead to a reduction in the quality of the opinions.

On the other hand, however, there is substantial knowledge on how to provide feedback and there is no single recipe. In some situations immediacy of feedback may yield feedback of higher quality (see e.g., Huang et al., 2008), in other situations terminal or delayed feedback may be found to be more effective (Walsh et al., 2009). Huet et al. (2009) showed that self-regulated concurrent feedback led to better learning outcomes (in pilot training) than imposed concurrent feedback. The timing of feedback should, therefore, be consistent with the recommendations flowing from research in a given area.

\subsection{Incremental feedback protects from creativity's going astray}

If the motto from IDEO (Kelley and Littman, 2001), a legendary design firm from Palo Alto, California, 'Fail often, in order to succeed sooner' is correct, there must be in place a system of incremental, stepwise correcting procedures, guiding the creative process. Feedback should be offered by the e-learning system in increments, in responses to small steps of the creative process, rather than offered only at the end (Qiu and Riesbeck, 2008). Consequently, it may generate substantial educational benefit, because students' failed attempts and misguided choices are corrected early, before time and resource commitment makes it psychologically difficult for a student to retrace steps and look for a better line of thought (see also Jonas et al., 2001).

Discarding ideas or prototypes which do not look promising is easy (in the psychological sense) at early stages and becomes progressively more difficult and costly at later stages, when a significant investment has been made. A well-known psychological and economic phenomenon known as 'Concord fallacy' shows that sunk costs may exert unduly strong influence on our decisions. A system of incremental feedback, when taken seriously, can prevent such fallacy from occurring (see also Lant and Hurley, 1999).

\subsection{Impartial and impersonal feedback removes peer pressure and hidden agendas}

The goal is to generate feedback that is free from favouritism or any form of bias. Such deviation from objectivity may result from the person's giving feedback having a stake in being either too critical or too uncritical with respect to the person being evaluated. Either form of departure from objectivity has a negative impact on the quality of feedback and, eventually, on the quality of the resulting solution.

Removing personal aspect from feedback protects from a broad spectrum of organisational and behavioural pathologies, such as sexism, ageism, racism and other forms of stereotyping and non-merit-based feedback. Even simple peer pressure 
originating from peer assessment may have negative impact on the quality of learning (Papinczak et al., 2007).

Pre-programmed online procedures may be equipped with appropriate filters (ideally working as editing tools in real time rather than as post hoc censorship), detecting language which indicates one of these forms of destructive feedback. Also, if they are equipped with a set of automated feedback statements, can be more efficient in this regard than any human assistance.

\section{A robust philosophy of evaluation in e-learning}

My main purpose here is to establish some fundamental rules of thought for developing a critical functionality in any e-learning system: a set of procedures used for evaluation, i.e., an assessment module. What will be evaluated and how to implement the procedures within the available IT environment are secondary issues. We need to clarify first why this particular aspect of e-learning is so important.

\subsection{No room for subjectivity and manipulation: make the criteria of quality explicit}

There are as many dimensions of quality as there are dimensions of human interest. Comprehensive software which can be used in as many contexts as possible should have a substantial base of optional criteria to choose from, with several measurement scales thought through, decided upon and prepared. It forces us to be clear and formulate our criteria for assessing the quality of solutions up-front, e.g., Garvin's (1987) eight quality dimensions in business (performance, features, reliability, conformance, durability, serviceability, aesthetics, and perceived quality), twenty core criteria for assessment of e-learning quality (see Bell and Farrier, 2007), or ten criteria applicable in the world of engineering (physico-mechanical, geometric, energetic, economic feasibility, design, degree of standardisation/unification, ergonomics and safety for the user, and reliability; Góralski, 1980).

Designers of e-learning systems should rely on the attributes that emphasise that such systems are: accessible, adaptable, administrable, affordable, agile, customisable, dependable, evolvable, extensible, flexible, learnable, mobile, modular, portable, predictable, simple, stable, understandable, and usable (source: Wikipedia). Ideally, such features should characterise e-learning systems simultaneously.

While some of the criteria may be limited in their applicability to software, others have universal and fundamental significance, such as simplicity, reliability or durability. Criteria in other fields may be similar, parallel in meaning, but may be termed differently, although we should beware a universal tendency of the authors overstating their assessment of the quality and significance of their own results (Jasieński, 2006, 2009).

\subsection{No room for hand-waving: make the criteria quantitative}

The sage admonishment from Galileo to 'measure what is measurable, and what is not measurable, make measurable' is the basis of the modern paradigm in management, known under the name of 'big data' (McAfee and Brynjolfsson, 2012). The benefits that 'big data' can bring may be derived from the development and utilisation of numerous 
indices and indicators quantifying basically any aspect of economic activity or of customers' or employees' behaviour. After all, each quantitative index represents a researcher's attempt at capturing the bewildering complexity of the phenomena in psychology, economics, biology etc.

Once the criteria have become quantitative, the users' responses accumulated by an IT system constitute data from which the shape of the distribution of individual scores and their correlation structure may be inferred. Such knowledge may be very useful in introducing various correcting factors, thus making the indices more comprehensive, more robust and less biased.

Similarly, mining data obtained from recording patterns of classroom or e-learning activity of students (using e.g., Moodle) may lead to successful clustering of students based on their cognitive styles (Jovanovic et al., 2012) or to building customised content recommendations for them (Khribi et al., 2009). In a sense, the wealth of data obtained from the users of an e-learning system constitutes, by itself, feedback (Khribi et al., 2009). Importantly, the more data we have, the more important it becomes to find good ways of simplifying and visualising them, which is best accomplished by a dedicated IT module.

The trend towards quantification, however, comes with new responsibilities, when we must understand and be ready to justify each of our methodological decisions. For example, the decision about the type of scale to use (e.g., interval-ordinal-nominal, Likert vs. continuous, arithmetic vs. logarithmic etc.) and about the precision with which the measurements are taken should be based on careful reasoning about the underlying process (physiological, psychological, microeconomic etc.) we are trying to capture in our analyses.

\subsection{No room for simple-mindedness: do not settle for unidimensional analyses}

The simplest explanation of this issue would be by comparing it to a simple regression analysis, but in which we include numerous $\mathrm{X}$ (independent) variables and several $\mathrm{Y}$ (dependent) variables. We feel that a complex problem requires applying a comprehensive (i.e., multivariate) approach. $\mathrm{X}$ variables are usually components (such as chemical substances mixed in various proportions) or factors (such as temperature, pressure, age, teacher's experience). The Y variables could be e.g., quality variables (see above) or physicochemical properties or some measures of educational or professional performance of teams or of individuals.

However, the decision of including many criteria of quality makes it more likely that variables measuring them will be correlated, i.e., assess very similar aspects of quality. Since each partial criterion would receive some weight, their combined contribution to the overall score would be excessive, thus biasing the total towards some particular aspects of quality. The difficulty of taking the correlation structure of the criteria into account arises from the fact that one does not know what the correlations are until a certain number of analyses have been performed.

One could limit the list of criteria to subsets revealed through cluster analysis or principal component analysis. However, it could be difficult for the users to evaluate abstract, derived criteria that would result from replacing simple, descriptive characteristics with principal components. Therefore, it may be preferable to retain these simple and intuitively more obvious criteria, however many there are, but then rely on quantitative analyses to reduce the number of dimensions. 


\subsection{No room for redundancy: make the model realistic by weighting}

With many criteria to be simultaneously analysed, there are important methodological decisions to be made; for example, when forming a single aggregate objective function, should the criteria receive equal weights? Probably not; as the result of weighting, some of the variables may become, for all practical purposes, meaningless, when their weights are set with sufficiently low values that their contribution to the numerical outcome of the entire ranking process is negligible.

For example, when e.g., costs are irrelevant for wealthy customers, the criterion 'cost' may be given the weight of 0.01 or may be skipped altogether. This is just one example of subjectivity that may greatly affect the outcome of the analysis. Importantly, decisions made when each factor is evaluated by individual subjects (participants, students etc.) are completely separate from the researcher's or administrator's decisions on weighting applied to each of the factors.

\subsection{No room for simple-mindedness: optimise multiple objectives}

Another challenge is to correctly localise the optimum, from the perspective of the desired set of criteria. Response surface methodology provides statistical tools to explore the landscape created e.g., by combinations of conditions set up by the experimenter or of variables measured by the researcher. As if this was not difficult enough, we often face the challenge of simultaneously optimising two or more conflicting objectives. Usually, trade-offs between objectives appear, which forces us to accept Pareto optimal solutions.

\subsection{No room for wishful thinking: justify decisions with statistics}

After having collected all the data, then a composite index can be calculated, but it means that statistical requirements must be taken into account (see below). The computational ease with which one can create new types of metrics does not provide any guarantee that such quantitative indices are accurate, precise, and possess appropriate statistical properties. For example, the ratio-based indices are characterised by very broad confidence intervals, thus making hypothesis testing difficult, if not impossible (see Jasieński and Bazzaz, 1999).

\subsection{No room for arbitrariness: justify decisions with sensitivity analyses}

Even a most comprehensive list of quality criteria, each evaluated in a quantitative manner, may suffer from a fundamental methodological weakness, namely that of arbitrariness. After all, decisions of which criteria to use, along which scale to evaluate them and how to weight their contributions to the overall score, may significantly impact the final selection of the best or most desirable solutions/ideas. The notion of arbitrariness in the selection or weighting of the criteria focuses our attention on the risk of making decisions which may be wrong because they are based on unrepresentative assumptions.

By 'unrepresentative' I mean such assumptions which are atypical, strange, unusual and do not correctly represent the overall range of available options. There is no reason to suggest that arbitrariness arises only from irrationality, whim, capriciousness or wilful manipulation aimed at influencing the result of analysis. Sometimes these assumptions may result from traditionally accepted rules of decision-making (e.g., a tradition of using 
certain indices rather than others, postulated by a competing academic school of thought) or from a sense of propriety (of what is acceptable or elegant).

Even when the selection of criteria is based on honest judgment, reason, or scientific principles, one could ask what would happen if another set of equally relevant inputs were to be used. Would the final conclusions/outputs be the same? To give a simple example: how is the aesthetic value of jewellery (or quality of gold-plated jacks in audiophile speakers) affected if we replace $30 \%$ of gold with copper? How would it change if we replace $50 \%$ ?

This is the basis of sensitivity analysis and involves assessing the degree to which the final outcome (output) depends on the initial assumptions or inputs (with respect to the particular criteria chosen, partial weights or scales etc.). Since these initial 'settings' are to some extent subjective, we can slightly modify the values assigned to each criterion and redo the computations. Some of the parameters may turn out to be more important than others, i.e., changes in their values (settings) may be more strongly reflected in the output. Also, the responses may be non-linear, i.e., may be relatively greater or smaller than the changes in the parameters. We are basically exploring the nearest vicinity of the parameter space of the chosen solution and testing the stability of this solution. If it is still the best, after having tested various combinations of the criteria, we may safely accept this solution as optimal.

\section{The assessment module: criteria of quality quantification}

In a comprehensive e-learning system which comprises, apart from other educational tools, several methods of creative thinking, a set of grading and evaluation procedures (for coursework or theses), a module comprising criteria for assessment may be built as a common resource to serve the needs of all methods. It should make it easy to use quantitative methods since it may have various built-in functionalities for computing and visualising indices, changing measurement scales, transforming the variables etc.

An integrated module should also be equipped with modern options for carrying out sensitivity analyses and for computer-intensive calculations (e.g., bootstrap-based methods) of statistical confidence limits in the case of non-standard quantitative metrics. Pre-programmed procedures should also have appropriate statistical analyses to take the correlation of the partial criteria into account.

The module may also be used for the purpose of course evaluation, after having selected from the available options the criteria of quality appropriate for assessment of educational skills, rather than creative content of ideas. An important benefit derived from applying online tools is reducing the degree of subjectivity and imprecision in evaluation which are both negative features of direct human interactions (see also: Smyrnova-Trybulska, 2009; Stodberg, 2012).

\section{The nominal group method: a case illustrating the importance of anonymity and feedback for creativity}

Organisational behaviour as a discipline has accumulated observations of various forms of social pathologies, such as groupthink and groupshift, which limit the quality of group-based thinking. The essence of the nominal group method is restraining or limiting 
peer-pressure, by introducing separation between two important stages of intellectual work (Figure 1): individual creative output of each participant and feedback and criticism from the group (Greenberg and Baron, 2000).

\subsection{Stages in the nominal group method}

First stage in this method involves providing participants with information about the topic of the debate. Participants should be protected from any form of social pressure and should be able to privately develop their ideas about solutions. Second stage involves making all ideas available online to the group of participants. The contributions should be shown in a non-partial, non-biased way, preferably with removed authorship information and with each suggested idea equally easily accessible for comments.

During the third stage, each idea is discussed and clarified by the participants, with comments and criticisms being visible to all members of the group, again preferably without authorship information. The virtual nature of 'group' exchange of views is clearly superior to the real meeting, because ensuring anonymity of comments is in reallife situation nearly impossible to accomplish. Withholding knowledge about the identity of the referees greatly increases the quality of comments and improves honesty of the evaluations (see earlier discussion on anonymity).

During the fourth stage numerical evaluation of ideas occurs, when the scores are submitted online (using the assessment module), according to the criteria established and made very explicit. Participants know only the content of each proposal and the criticisms it has accumulated during the third stage, and based on this information must privately make decisions about numerical scores to assign to each proposal. The outcomes of the scoring stage are a private ranking of all proposals that is submitted by each participant and that reflects his or her order of preference. In order to decide on a single ranking of all proposals, all individually-assigned ranks are averaged and the highest-ranking (on the average) idea is taken as the group's decision.

Figure 1 A flowchart of the nominal group method as implemented online

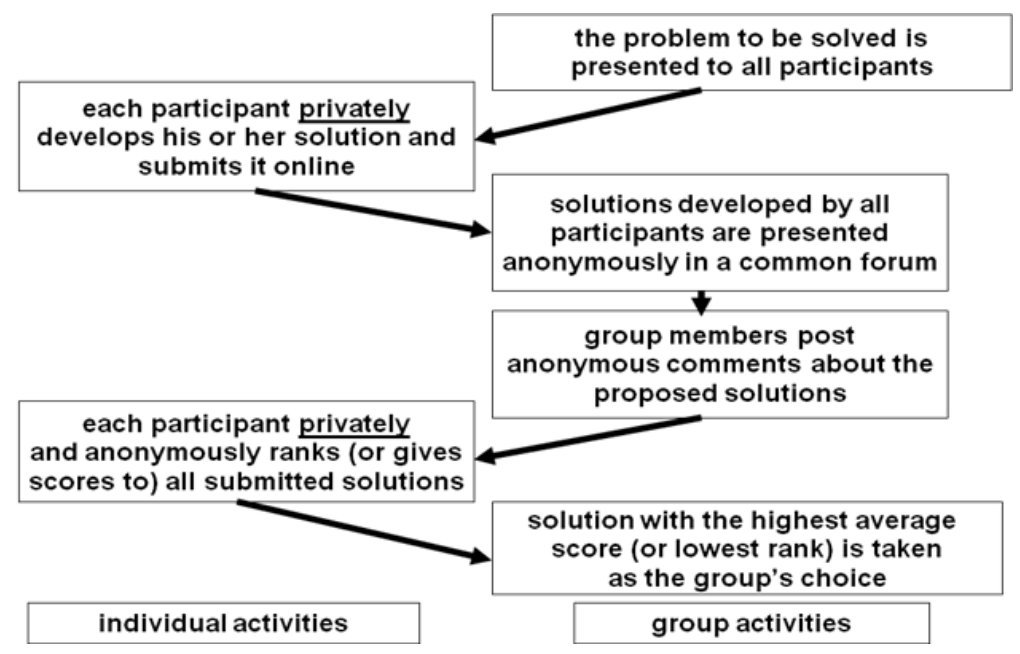

Source: Own elaboration, modified from a flowchart of a traditional version presented by Greenberg and Baron (2000, p.359). 


\subsection{Benefits of an e-learning implementation}

Online systems can very effectively accomplish the goal of removing face-to-face peer pressure. Individual contributions are anonymised during the idea-generation and idea-evaluation stages, but the authorship of the best ideas may be revealed at the end of the procedure to ensure recognition of the creative employees. Furthermore, integrating the module dedicated to the nominal group method with the assessment module enables precise and quantitative ranking of ideas.

\section{Conclusions}

Many people are freed now from constraints in access to information, knowledge and wisdom of others. Thanks to such egalitarian resources as Wikipedia, chances of various groups of users, e.g., of rural population or of disabled individuals, are equalised (other things being equal, of course). Similarly, people, whose development is constrained by a political regime or social and religious norms or organisational culture at their workplace, may be helped by technology in realising their full potential.

However, lack of limitations in access to information and structured content form a necessary, but not a sufficient condition of good educational standards. There must be an appropriately nurturing environment, created, in part, by our IT-based tools. They may be invaluable in managing creativity of ideas and fostering independence of thought. They may also teach rational thinking by forcing the users to be precise and rely on explicitly defined criteria. Leaving young people without guidance and clear, unbiased assessment, lost in the labyrinth of overabundance of images, ideas, and trivia, would mean not fulfilling the mission of teaching them how to think critically and creatively.

\section{Acknowledgements}

I was supported by the European Union under the Marie Curie Industry-Academia Partnership and Pathways programme (IAPP) - project number 324448 'Reinvent: Transforming SMEs in creative sectors through business model innovation'. I also thank the 'Creator of Innovativeness' programme of the Polish National Center for Research and Development (NCBiR). I thank Tomasz Golab, Magdalena Rzeznik, and Artur Stawiarski for numerous discussions during which many of the ideas discussed here have crystallised.

\section{References}

Amabile, T. (1998) 'How to kill creativity', Harvard Business Review, September-October, Vol. 76, No. 5, pp.77-87.

Anwar, M. and Greer, J. (2012) 'Facilitating trust in privacy-preserving e-learning environments', IEEE Transactions on Learning Technologies, Vol. 5, No. 1, pp.62-73.

Bell, M. and Farrier, S. (2007) 'Measuring success in e-learning - a multi-dimensional approach', in 6th European Conference on E-Learning, pp.43-50. 
Boaler, J., Altendorff, L. and Kent, G. (2011) 'Mathematics and science inequalities in the United Kingdom: when elitism, sexism and culture collide', Oxford Review of Education, Vol. 37, No. 4, pp.457-484.

Butler, D.L. and Winne, P.H. (1995) 'Feedback and self-regulated learning - a theoretical synthesis', Review of Educational Research, Vol. 65, No. 3, pp.245-281.

Chang, C.M. (2011) 'New organizational designs for promoting creativity: a case study of virtual teams with anonymity and structured interactions', Journal of Engineering and Technology Management, Vol. 28, No. 4, pp.268-282.

Chi, Y.L. and Chen, C.Y. (2009) 'Project teaming: knowledge-intensive design for composing team members', Expert Systems with Applications, Vol. 36, No. 5, pp.9479-9487.

Daly, S.R., Yilmaz, S., Christian, J.L., Seifert, C.M. and Gonzalez, R. (2012) 'Design heuristics in engineering concept generation', Journal of Engineering Education, Vol. 101, No. 4, pp.601-629.

Davila, T., Epstein, M. and Shelton, R. (2005) Making Innovation Work: How to Manage It, Measure It, and Profit from It, Wharton School Publishing, Upper Saddle River.

de Bono, E. (1992) Serious Creativity. Using the Power of Lateral Thinking to Create New Ideas, HarperBusiness, New York.

Deming, W.E. (1982) Out of the Crisis, MIT Press, Cambridge.

Garvin, D.A. (1987) 'Competing on the eight dimensions of quality', Harvard Business Review, Vol. 65, No. 6, pp.101-109.

Gilbert, J., Morton, S. and Rowley, J. (2007) 'E-learning: the student experience', British Journal of Educational Technology, Vol. 38, No. 4, pp.560-573.

Góralski, A. (1980) Creative Problem-Solving, PWN, Warszawa (in Polish).

Greenberg, J. and Baron, R.A. (2000) Behavior in Organizations, Prentice Hall, Upper Saddle River.

Handley, K. and Williams, L. (2011) 'From copying to learning: using exemplars to engage students with assessment criteria and feedback', Assessment \& Evaluation in Higher Education, Vol. 36, No. 1, pp.95-108.

Huang, C.J., Chen, C.H., Luo, Y.C., Chen, H.X. and Chuang, Y.T. (2008) 'Developing an intelligent diagnosis and assessment e-learning tool for introductory programming', Educational Technology \& Society, Vol. 11, No. 4, pp.139-157.

Huet, M., Jacobs, D.M., Camachon, C., Goulon, C. and Montagne, G. (2009) 'Self-controlled concurrent feedback facilitates the learning of the final approach phase in a fixed-base flight simulator', Human Factors, Vol. 51, No. 6, pp.858-871.

Jara, M. and Mellar, H. (2010) 'Quality enhancement for e-learning courses: the role of student feedback', Computers \& Education, Vol. 54, No. 3, pp.709-714.

Jasieński, M. (2006) 'It's incredible how often we're surprised by findings', Nature, Vol. 440, No. 7088, p.1112.

Jasieński, M. (2007) 'Theory-free and fact-free but method-focused and trust-driven education: insights from Google, Excel, and eBay', in 5th International Conference on Technology in Teaching and Learning in Higher Education, Nowy Sącz, pp.39-44.

Jasieński, M. (2009) 'Garfield's demon and 'surprising' or 'unexpected' results in science', Scientometrics, Vol. 78, No. 2, pp.347-353.

Jasieński, M. and Bazzaz, F.A. (1999) 'The fallacy of ratios and testability of models in biology', Oikos, Vol. 84, No. 2, pp.321-326.

Jasieński, M. and Rzeźnik, M. (2012) 'Innovatics - a new toolbox of skills for innovative production managers', in Knosala, R. (Ed.): Innovations in Management and Production Engineering, pp.63-71, Polish Association for Production Management, Opole. 
Jonas, E., Frey, D., Henninger, M., Pommer, M., Schulz-Hardt, S., von Haeften, I. and Mandl, H. (2001) 'Explaining one's own behavior as a factor influencing the search for and evaluation of information in a sequential learning situation', Zeitschrift für Entwicklungspsychologie und Pädagogische Psychologie, Vol. 33, No. 4, pp.242-252.

Jovanovic, M., Vukicevic, M., Milovanovic, M. and Minovic, M. (2012) 'Using data mining on student behavior and cognitive style data for improving e-learning systems: a case study', International Journal of Computational Intelligence Systems, Vol. 5, No. 3, pp.597-610.

Kelley, T. and Littman, J. (2001) The Art of Innovation. Lessons in Creativity from IDEO, America's Leading Design Firm, Crown Business, New York.

Khribi, M.K., Jemni, M. and Nasraoui, O. (2009) 'Automatic recommendations for e-learning personalization based on web usage mining techniques and information retrieval', Educational Technology \& Society, Vol. 12, No. 4, pp.30-42.

Krass, D. and Ovchinnikov, A. (2006) 'The University of Toronto's Rotman School of Management uses management science to create MBA study groups', Interfaces, Vol. 36, No. 2, pp.126-137.

Krause, U.M., Stark, R. and Mandl, H. (2009) 'The effects of cooperative learning and feedback on e-learning in statistics', Learning and Instruction, Vol. 19, No. 2, pp.158-170.

Lant, T.K. and Hurley, A.E. (1999) 'A contingency model of response to performance feedback escalation of commitment and incremental adaptation in resource investment decisions', Group \& Organization Management, Vol. 24, No. 4, pp.421-437.

Lewis, N.J. and Orton, P. (2000) 'The five attributes of innovative e-learning', Training \& Development, Vol. 54, No. 6, pp.47-51.

Macfadyen, L.P. and Dawson, S. (2012) 'Numbers are not enough. Why e-learning analytics failed to inform an institutional strategic plan', Educational Technology \& Society, Vol. 15, No. 3 , pp.149-163.

McAfee, A. and Brynjolfsson, E. (2012) 'Big data: the management revolution', Harvard Business Review, Vol. 90, No. 10, pp.60-68.

Mockus, J. (2010) 'On examples of e-education environment for distance graduate studies', Computer Applications in Engineering Education, Vol. 18, No. 2, pp.331-343.

Nicol, D.J. and Macfarlane-Dick, D. (2006) 'Formative assessment and self-regulated learning: a model and seven principles of good feedback practice', Studies in Higher Education, Vol. 31, No. 2, pp.199-218.

Palmer, S. and Holt, D. (2010) 'Students' perceptions of the value of the elements of an online learning environment: looking back in moving forward', Interactive Learning Environments, Vol. 18, No. 2, pp.135-151.

Papinczak, T., Young, L. and Groves, M. (2007) 'Peer assessment in problem-based learning: a qualitative study', Advances in Health Sciences Education, Vol. 12, No. 2, pp.169-186.

Qiu, L. and Riesbeck, C.K. (2008) 'Human-in-the-loop: a feedback-driven model for authoring knowledge-based interactive learning environments', Journal of Educational Computing Research, Vol. 38, No. 4, pp.469-509.

Smyrnova-Trybulska, E. (2009) 'On principles of the design and assessment of distance courses', Distance Learning, Simulation and Communication 2009, Proceedings, pp.159-164.

Smyrnova-Trybulska, E. and Grudzień, J. (2009) 'E-learning in the computer science: some computer and methodology techniques', The New Educational Review, Vol. 19, Nos. 3-4, pp.147-161.

Smyrnova-Trybulska, E. and Stach, S. (Eds.) (2012) Using LCMS Moodle as a System for Distance Learning Support, p.560, Studio-Noa, University of Silesia, Katowice (in Polish).

Stach, S. and Smyrnova-Trybulska, E. (Eds.) (2012) The Use of CMS Systems in Developing the Information and Education Environments on the Internet, p.204, Studio-Noa, University of Silesia, Katowice (in Polish).

Stodberg, U. (2012) 'A research review of e-assessment', Assessment \& Evaluation in Higher Education, Vol. 37, No. 5, pp.591-604. 
Thoms, B. (2011) 'A dynamic social feedback system to support learning and social interaction in higher education', IEEE Transactions on Learning Technologies, Vol. 4, No. 4, pp.340-352.

Torr, G. (2008) Managing Creative People: Lessons in Leadership for the Ideas Economy, Wiley, Chichester.

Trompenaars, F. (2007) Riding the Whirlwind: Connecting People and Organisations in a Culture of Innovation, Infinite Ideas Limited, Oxford.

Tsai, C.W. (2010) 'The effects of feedback in the implementation of web-mediated self-regulated learning', Cyberpsychology Behavior and Social Networking, Vol. 13, No. 2, pp.153-158.

Walsh, C.M., Ling, S.C., Wang, C.S. and Carnahan, H. (2009) 'Concurrent versus terminal feedback: it may be better to wait', Academic Medicine, Vol. 84, No. 10, pp.S54-S57.

Yong, J.M. (2008) 'Enhancing the privacy of e-learning systems with alias and anonymity', Computer Supported Cooperative Work in Design IV, Lecture Notes in Computer Science, Vol. 5236, pp.534-544. 